\title{
Iterative Coupling of Mixed and Discontinuous Galerkin Methods for Poroelasticity
}

\author{
Markus Bause \\ Helmut Schmidt University, Faculty of Mechanical Engineering, \\ Holstenhofweg 85, 220433 Hamburg, Germany
}

\begin{abstract}
We analyze an iterative coupling of mixed and discontinuous Galerkin methods for numerical modelling of coupled flow and mechanical deformation in porous media. The iteration is based on an optimized fixedstress split along with a discontinuous variational time discretization. For the spatial discretization of the subproblem of flow mixed finite element techniques are applied. The discretization of the subproblem of mechanical deformation uses discontinuous Galerkin methods. They have shown their ability to eliminate locking that sometimes arises in numerical algorithms for poroelasticity and causes nonphysical pressure oscillations.
\end{abstract}

\section{Introduction and mathematical model}

We consider the quasi-static Biot system of flow in deformable porous media,

$$
\begin{aligned}
& -\nabla \cdot(2 \mu \varepsilon(\boldsymbol{u})+\lambda \nabla \cdot \boldsymbol{u I}-b p \boldsymbol{I})=\rho_{b} \boldsymbol{g}, \\
& \partial_{t}\left(\frac{1}{M} p+\nabla \cdot(b \boldsymbol{u})\right)+\nabla \cdot \boldsymbol{q}=f, \quad \boldsymbol{q}=-\boldsymbol{K} \nabla p,
\end{aligned}
$$

in $\Omega \times I$ for a bounded Lipschitz domain $\Omega \subset \mathbb{R}^{d}$, with $d=2,3$, and $I=(0, T]$. For simplicity, it is supplemented by homogeneous initial and Dirichlet boundary conditions for $p$ and $\boldsymbol{u}$. We denote by $\boldsymbol{u}$ the unknown displacement field, $\boldsymbol{\varepsilon}(\boldsymbol{u})=\left(\nabla \boldsymbol{u}+(\nabla \boldsymbol{u})^{\top}\right) / 2$ the linearized strain tensor, $p$ the unknown fluid pressure, $\mu$ and $\lambda$ the Lamé constants, $b$ Biot's coefficient, $\rho_{b}$ the bulk density, $M$ Biot's modulus, $\boldsymbol{q}$ Darcy's velocity and $\boldsymbol{K}$ the permeability field. Further, $\boldsymbol{g}$ denotes gravity or, in general, some body force and $f$ is a volumetric source. We assume that $\boldsymbol{g}(0)=\mathbf{0}$. The quantities $\mu, \lambda, b, \rho_{b}$ and $M$ are positive constants. The matrix $\boldsymbol{K}$ is supposed to be symmetric and uniformly positive definite. Well-posedness of (1.1), (1.2) has been shown in the literature.

Recently, iterative coupling schemes for solving the Biot system (1.1), (1.2) have attracted researchers' interest and have shown their efficiency; cf., e.g., 1, 2, 3, and the references therein. Iterative coupling offers the appreciable advantage over the fully coupled method that existing and highly developed discretizations and algebraic 
solver technologies can be reused. Here, we use an "optimized fixed-stress split" type iterative method; cf. [1, 2, 3]. For the time discretization of the arising subproblems of flow and mechanical deformation a discontinuous in time variational approach (cf. [1]) is applied.

For the approximation of the pressure and flux unknown $p$ and $\boldsymbol{q}$ within the iterative coupling approach we use a mixed finite element method. The displacement variable $\boldsymbol{u}$ is discretized by a discontinuous Galerkin method. Thereby the results herein represent the natural extension of the work of the author in [1] and further work in the literature, where the displacement field $\boldsymbol{u}$ was approximated by a continuous Galerkin method. The motivation for using a discontinuous Galerkin scheme for the discretization of the displacement $\boldsymbol{u}$ comes from combating the locking phenomenon, that sometimes arises in numerical algorithms for poroelasticity and manifests as spurious nonphysical pressure oscillations. In poroelasticity the locking-dominant parameter is the specific storage coefficient $c_{0}=1 / M$ in Eq. (1.2). Locking primarily arises if $c_{0}=0$ and, usually, does not appear for $c_{0} \neq 0$. For a more extensive discussion of locking in poroelasticity we refer to the literature; cf., e.g., [5, 6].

In 4 a coupling of mixed and discontinuous Galerkin finite methods is studied within a monolithic approach and an error analysis for the semi-discretization is space is given. By numerical experiments it is shown for the problem of Barry and Mercer, that a discontinuous Galerkin discretization of the displacement $\boldsymbol{u}$ is capable of eliminating spurious pressure oscillations related to locking arising in continuous discretizations of $\boldsymbol{u}$. Therefore, it seems worth to study the combined mixed and discontinuous Galerkin approach also within a fixed-stress split iterative method which is done here.

We use standard notation. In particular, we put $W=L^{2}(\Omega)$ and $\boldsymbol{V}=\boldsymbol{H}(\operatorname{div} ; \Omega)$ and denote by $\langle\cdot, \cdot\rangle$ the inner product of $W$.

\section{Iterative coupling and space-time discretization}

We consider solving the system (1.1), (1.2) by the following iteration scheme.

Subproblem of flow: Let $f^{k}:=f-b \nabla \cdot \partial_{t} \boldsymbol{u}^{k}+L \partial_{t} p^{k} \in L^{2}(I ; W)$ be given. Find $p^{k+1} \in H^{1}(I ; W), \boldsymbol{q}^{k+1} \in L^{2}(I ; \boldsymbol{V})$ such that $p^{k+1}(0)=0$ and

$$
\begin{aligned}
& \left(\frac{1}{M}+L\right) \int_{I}\left\langle\partial_{t} p^{k+1}, w\right\rangle \mathrm{d} t+\int_{I}\left\langle\nabla \cdot \boldsymbol{q}^{k+1}, w\right\rangle \mathrm{d} t=\int_{I}\left\langle f^{k}, w\right\rangle \mathrm{d} t, \\
& \int_{I}\left\langle\boldsymbol{K}^{-1} \boldsymbol{q}^{k+1}, \boldsymbol{v}\right\rangle \mathrm{d} t-\int_{I}\left\langle p^{k+1}, \nabla \cdot \boldsymbol{v}\right\rangle \mathrm{d} t=0
\end{aligned}
$$

for all $w \in L^{2}(I ; W)$ and $\boldsymbol{v} \in L^{2}(I ; \boldsymbol{V})$.

Subproblem of mechanical deformation: Let $p^{k+1} \in H^{1}(I ; W)$ be given. Find $\boldsymbol{u}^{k+1} \in H^{1}\left(I ; \boldsymbol{H}^{1}(\Omega)\right) \cap L^{2}\left(I ; \boldsymbol{H}_{0}^{1}(\Omega)\right)$ such that $\boldsymbol{u}(0)=\mathbf{0}$ and

$$
\begin{gathered}
\int_{I} 2 \mu\left\langle\boldsymbol{\varepsilon}\left(\boldsymbol{u}^{k+1}\right), \boldsymbol{\varepsilon}(\boldsymbol{z})\right\rangle \mathrm{d} t+\int_{I} \lambda\left\langle\nabla \cdot \boldsymbol{u}^{k+1}, \nabla \cdot \boldsymbol{z}\right\rangle \mathrm{d} t \\
=\rho_{b} \int_{I}\langle\boldsymbol{g}, \boldsymbol{z}\rangle \mathrm{d} t+b \int_{I}\left\langle p^{k+1}, \nabla \cdot \boldsymbol{z}\right\rangle \mathrm{d} t
\end{gathered}
$$

for all $\boldsymbol{z} \in L^{2}\left(I ; \boldsymbol{H}_{0}^{1}(\Omega)\right)$. 
In this scheme the artificial quantity $L$ is a numerical parameter that was firstly introduced in [3] to accelerate the iteration process. The convergence of the iteration (2.1) - (2.3) is ensured for all $L \geq b^{2} /(2 \lambda)$; cf. [1, Thm. 2.1].

For the discretization we decompose the time interval $(0, T]$ into $N$ subintervals $I_{n}=\left(t_{n-1}, t_{n}\right]$, where $n \in\{1, \ldots, N\}$ and $0=t_{0}<t_{1}<\cdots<t_{N-1}<t_{N}=T$ and $\tau=\max _{n=1, \ldots N}\left(t_{n}-t_{n-1}\right)$. We denote by $\mathcal{T}_{h}=\{K\}$ a finite element decomposition of mesh size $h$ of the polyhedral domain $\bar{\Omega}$ into closed subsets $K$, quadrilaterals in two dimensions and hexahedrals in three dimensions. Further, $\mathcal{E}_{h}^{\text {int }}$ is the set of all interior edges (faces for $d=3$ ). To each interior edge (or face) $e \in \mathcal{E}_{h}^{\text {int }}$ we associate a fixed unit normal vector $\boldsymbol{\nu}^{e}$.

For the spatial discretization of (2.1), (2.2) we use a mixed finite element approach. We choose the class of Raviart-Thomas elements for the two-dimensional case and the class of Raviart-Thomas-Nédélec elements in three space dimensions, where for $s \geq 0$ the space $W_{h}^{s} \subset W$ with $W_{h}^{s}=\left\{w_{h} \in L^{2}(\Omega) \mid w_{\left.h\right|_{K}} \circ T_{K} \in \mathbb{Q}_{s}\right\}$ and $\boldsymbol{V}_{h}^{s} \subset \boldsymbol{V}$ denote the corresponding inf-sup stable pair of finite element spaces; cf. [1]. Here, $\mathbb{Q}_{s}$ is the space of polynomials that are of degree less than or equal to $s$ in each variable and $T_{K}$ is a suitable invertible mapping of the reference cube $\widehat{K}$ to $K$ of $\mathcal{T}_{h}$. For the spatial discretization of (2.3) we discretize we use a discontinuous Galerkin method with the space

$$
\boldsymbol{H}_{h}^{l}=\left\{\boldsymbol{z}_{h} \in L^{2}(\Omega) \mid \boldsymbol{z}_{\left.h\right|_{K}} \circ T_{K} \in \mathbb{Q}_{l}^{d}, \boldsymbol{z}_{h \mid \partial \Omega}=\mathbf{0}\right\} .
$$

The fully discrete space-time finite element spaces are then defined by

$$
\begin{aligned}
& \mathcal{W}_{\tau, h}^{r, s}=\left\{w_{\tau, h} \in L^{2}(I ; W) \mid w_{\tau, h \mid I_{n}} \in \mathcal{P}_{r}\left(I_{n} ; W_{h}^{s}\right), w_{\tau, h}(0) \in W_{h}^{s}\right\} \\
& \mathcal{V}_{\tau, h}^{r, s}=\left\{\boldsymbol{v}_{\tau, h} \in L^{2}(I ; \boldsymbol{V}) \mid \boldsymbol{v}_{\tau, h \mid I_{n}} \in \mathcal{P}_{r}\left(I_{n} ; \boldsymbol{V}_{h}^{s}\right), \boldsymbol{v}_{\tau, h}(0) \in \boldsymbol{V}_{h}^{s}\right\} \\
& \mathcal{Z}_{\tau, h}^{r, l}=\left\{\boldsymbol{z}_{\tau, h} \in L^{2}\left(I ; \boldsymbol{L}^{2}(\Omega)\right) \mid \boldsymbol{z}_{\tau, h \mid I_{n}} \in \mathcal{P}_{r}\left(I_{n} ; \boldsymbol{H}_{h}^{l}\right), \boldsymbol{z}_{\tau, h}(0) \in \boldsymbol{H}_{h}^{l}\right\},
\end{aligned}
$$

where $\mathcal{P}_{r}\left(I_{n} ; X\right)$ is the space of all polynomials in time up to degree $r \geq 0$ on $I_{n}$ with values in $X$. We choose $l=s+1$ to equilibrate the convergence rates of the spatial discretization for the three unknowns $p, \boldsymbol{q}$ and $\boldsymbol{u}$; cf. [4, p. 426, Thm. 1]. For short, we put $W_{h}=W_{h}^{s}, \boldsymbol{V}_{h}=\boldsymbol{V}_{h}^{s}$ and $\boldsymbol{H}_{h}=\boldsymbol{H}_{h}^{s+1}$.

On each time interval $I_{n}$ we expand the discrete functions $p_{\tau, h}^{k} \in \mathcal{W}_{\tau, h}^{r, s}, \boldsymbol{q}_{\tau, h}^{k} \in \mathcal{V}_{\tau, h}^{r, s}$ and $\boldsymbol{u}_{\tau, h}^{k} \in \mathcal{Z}_{\tau, h}^{r, s+1}$ in time in terms of Lagrangian basis functions $\varphi_{n, j} \in \mathcal{P}_{r}\left(I_{n} ; \mathbb{R}\right)$ with respect to $r+1$ nodal points $t_{n, j} \in I_{n}$, for $j=0, \ldots, r$,

$$
p_{\tau, h \mid I_{n}}^{k}(t)=\sum_{j=0}^{r} P_{n, h}^{j, k} \varphi_{n, j}(t), \quad \boldsymbol{z}_{\tau, h \mid I_{n}}^{k}(t)=\sum_{j=0}^{r} \boldsymbol{Z}_{n, h}^{j, k} \varphi_{n, j}(t),
$$

for $t \in I_{n}$ and $\boldsymbol{z} \in\{\boldsymbol{q}, \boldsymbol{u}\}$ with coefficient functions $P_{n, h}^{j, k} \in W_{h}, \boldsymbol{Q}_{n, h}^{j, k} \in \boldsymbol{V}_{h}$ for $\boldsymbol{z}=\boldsymbol{q}$ and $\boldsymbol{U}_{n, h}^{j, k} \in \boldsymbol{H}_{h}$ for $\boldsymbol{z}=\boldsymbol{u}$ and $j=0, \ldots, r$. The nodal points $t_{n, j}$ are chosen as the quadrature points of the $(r+1)$-point Gauss quadrature formula on $I_{n}$ which is exact for polynomials of degree less or equal to $2 r+1$.

Solving the variational problems (2.1), (2.2) and (2.3) in the fully discrete function spaces (2.4)-(2.6) and using a discontinuous test basis in time with support on 
$I_{n}$ then leads us to the following fully discrete iteration scheme, referred to as the $\operatorname{MFEM}(s) \mathrm{dG}(s+1)-\mathrm{dG}(r)$ splitting scheme.

Fully discrete subproblem of flow: Let $n \in\{1, \ldots, N\}$. Find coefficient functions $P_{n, h}^{i, k+1} \in W_{h}$ and $\boldsymbol{Q}_{n, h}^{i, k+1} \in \boldsymbol{V}_{h}$ for $i=0, \ldots, r$ such that

$$
\begin{aligned}
& \frac{1}{M} \sum_{j=0}^{r} \alpha_{i j}\left\langle P_{n, h}^{j, k+1}, w_{h}\right\rangle+L \sum_{j=0}^{r} \alpha_{i j}\left\langle P_{n, h}^{j, k+1}-P_{n, h}^{j, k}, w_{h}\right\rangle+\tau_{n} \beta_{i i}\left\langle\nabla \cdot \boldsymbol{Q}_{n, h}^{i, k+1}, w_{h}\right\rangle \\
& =\tau_{n} \beta_{i i}\left\langle f\left(t_{n, i}\right), w_{h}\right\rangle-b \sum_{j=0}^{r} \alpha_{i j} \sum_{K \in \mathcal{T}_{h}}\left\langle\nabla \cdot \boldsymbol{U}_{n, h}^{j, k}, w_{h}\right\rangle_{K} \\
& +\gamma_{i} \frac{1}{M}\left\langle p_{\tau, h}^{\infty}\left(t_{n-1}^{-}\right), w_{h}\right\rangle+\gamma_{i} b \sum_{K \in \mathcal{T}_{h}}\left\langle\nabla \cdot \boldsymbol{u}_{\tau, h}^{\infty}\left(t_{n-1}^{-}\right), w_{h}\right\rangle_{K} \\
& \left\langle\boldsymbol{K}^{-1} \boldsymbol{Q}_{n, h}^{i, k+1}, \boldsymbol{v}_{h}\right\rangle-\left\langle P_{n, h}^{i, k+1}, \nabla \cdot \boldsymbol{v}_{h}\right\rangle=0
\end{aligned}
$$

for all $w_{h} \in W_{h}, \boldsymbol{v}_{h} \in \boldsymbol{V}_{h}$ and $i=0, \ldots, r$, where $p_{\tau, h}\left(t_{n-1}^{-}\right)=0$ for $n=1$ and $p_{\tau, h}^{\infty}\left(t_{n-1}^{-}\right)=\lim _{k \rightarrow \infty} p_{\tau, h}^{k}\left(t_{n-1}^{-}\right)$for $n>1$, and similarly for $\boldsymbol{u}_{\tau, h}^{\infty}\left(t_{n-1}^{-}\right)$.

Fully discrete subproblem of mechanical deformation: Let $n \in\{1, \ldots, N\}$. Find coefficient functions $\boldsymbol{U}_{n, h}^{i, k+1} \in \boldsymbol{H}_{h}$ for $i=0, \ldots, r$ such that

$$
\begin{aligned}
& \sum_{K \in \mathcal{T}_{h}}\left\langle\boldsymbol{\sigma}\left(\boldsymbol{U}_{n, h}^{i, k+1}\right), \boldsymbol{\varepsilon}\left(\boldsymbol{z}_{h}\right)\right\rangle_{K}+J_{\delta}\left(\boldsymbol{U}_{n, h}^{i, k+1}, \boldsymbol{z}_{h}\right)-J_{d}\left(\boldsymbol{\sigma}\left(\boldsymbol{U}_{n, h}^{i, k+1}\right), \boldsymbol{z}_{h}\right) \\
& =\sum_{K \in \mathcal{T}_{h}} b\left\langle P_{n, h}^{i, k+1}, \nabla \cdot \boldsymbol{z}_{h}\right\rangle_{K}-b J_{p}\left(P_{n, h}^{i, k+1}, \boldsymbol{z}_{h}\right)
\end{aligned}
$$

for all $\boldsymbol{z}_{h} \in \boldsymbol{H}_{h}$ and $i=0, \ldots, r$ with effective stress $\boldsymbol{\sigma}(\boldsymbol{u})=2 \mu \boldsymbol{\varepsilon}(\boldsymbol{u})+\lambda \nabla \cdot \boldsymbol{u} \boldsymbol{I}$.

In Eq. (2.10) we use the notation

$$
\begin{aligned}
& J_{\delta}\left(\boldsymbol{y}_{h}, \boldsymbol{z}_{h}\right)=\sum_{e \in \mathcal{E}_{h}^{\text {int }}} \frac{\delta_{e}}{|e|^{\beta}}\left\langle\left[\boldsymbol{y}_{h}\right],\left[\boldsymbol{z}_{h}\right]\right\rangle_{e}, \quad J_{p}\left(w_{h}, \boldsymbol{z}_{h}\right)=\sum_{e \in \mathcal{E}_{h}^{\text {int }}}\left\langle\left\{w_{h}\right\} \boldsymbol{\nu}^{e},\left[\boldsymbol{z}_{h}\right]\right\rangle_{e}, \\
& J_{d}\left(\boldsymbol{y}_{h}, \boldsymbol{z}_{h}\right)=\sum_{e \in \mathcal{E}_{h}^{\text {int }}}\left(\left\langle\left\{\boldsymbol{\sigma}\left(\boldsymbol{y}_{h}\right) \boldsymbol{\nu}^{e}\right\},\left[\boldsymbol{z}_{h}\right]\right\rangle_{e}+\left\langle\left\{\boldsymbol{\sigma}\left(\boldsymbol{z}_{h}\right) \boldsymbol{\nu}^{e}\right\},\left[\boldsymbol{y}_{h}\right]\right\rangle_{e}\right) .
\end{aligned}
$$

We denote by $\langle\cdot, \cdot\rangle_{K}$ and $\langle\cdot, \cdot\rangle_{e}$ the $L^{2}$ inner products on $K$ and $e$, respectively. As usual, we let $\{w\}=\left(\left(w_{\mid K}\right)_{\mid e}+\left(w_{\mid K^{\prime}}\right)_{\mid e}\right) / 2$ for two adjacent elements $K$ and $K^{\prime}$ with common edge (or face) $e$ and, similarly, $[w]=\left(w_{\mid K}\right)_{\mid e}-\left(w_{\mid K^{\prime}}\right)_{\mid e}$. The penalty term $J_{\delta}$ contains the numerical parameter $\delta_{e}$ that takes a constant value at each edge (or face) $e \in \mathcal{E}_{h}^{\text {int }}$ with Lebesgue measure $|e|$. The power $\beta$ is a positive number that depends on the dimension $d$. In [4, the choice $\beta=(d-1)^{-1}$ is proposed for the fully coupled semi-discretization of (1.1), (1.2). The coefficients $\alpha_{i j}, \beta_{i i}$ and $\gamma_{i}$ are defined by $\alpha_{i j}=\int_{I_{n}} \varphi_{n, j}^{\prime}(t) \cdot \varphi_{n, i}(t) \mathrm{d} t+\gamma_{i} \cdot \gamma_{j}, \beta_{i i}=\int_{I_{n}} \varphi_{n, i}(t) \cdot \varphi_{n, i}(t) \mathrm{d} t$ and $\gamma_{i}=\varphi_{n, i}\left(t_{n-1}^{+}\right)$ for $i, j=0, \ldots, r$. For a detailed derivation of the Eqs. (2.8)-(2.10) we refer to [1] for the space-time issue and to [4] for the discontinuous Galerkin approximation in space 
of the displacement field $\boldsymbol{u}$. Eq. (2.10) is referred to as the symmetric interior penalty (SIP) discontinuous Galerkin method. Finally, we note that an additional penalty term involving $\partial_{t} \boldsymbol{u}$ is proposed for the semi-discrete fully coupled approach in [4].

\section{Convergence of the iteration scheme}

Here we prove the convergence of the iteration scheme (2.8), (2.9) and (2.10). For this, let $\left\{p_{\tau, h}, \boldsymbol{q}_{\tau, h}\right\} \in \mathcal{W}_{\tau, h}^{r, s} \times \mathcal{V}_{\tau, h}^{r, s}, \boldsymbol{u}_{\tau, h} \in \mathcal{Z}_{\tau, h}^{r, s+1}$ denote the fully discrete $\operatorname{MFEM}(s) \mathrm{dG}(s+1)$ $\mathrm{dG}(r)$ approximation of (1.1), (1.2), formally given by passing to the limit $k \rightarrow \infty$ in the scheme (2.8)-(2.10). Analogously to (2.7), let $\left\{p_{\tau, h}, \boldsymbol{q}_{\tau, h}, \boldsymbol{u}_{\tau, h}\right\}$ on $I_{n}$ be represented by coefficients $P_{n, h}^{j} \in W_{h}, \boldsymbol{Q}_{n, h}^{j} \in \boldsymbol{V}_{h}$ and $\boldsymbol{U}_{n, h}^{j} \in \boldsymbol{H}_{h}$ for $j=0, \ldots, r$. For $n \in\{1, \ldots, N\}$ and $t \in I_{n}$ we put

$$
E_{p}^{j, k}=P_{n, h}^{j, k}-P_{n, h}^{j}, \quad e_{p}^{k}(t)=\sum_{j=0}^{r} E_{p}^{j, k} \varphi_{n, j}(t), \quad S_{p}^{i, k}=\sum_{j=0}^{r} \alpha_{i j} E_{p}^{j, k} .
$$

The quantities $\boldsymbol{E}_{\boldsymbol{q}}^{j, k}, \boldsymbol{e}_{\boldsymbol{q}}^{k}, \boldsymbol{E}_{\boldsymbol{u}}^{j, k}, \boldsymbol{e}_{\boldsymbol{u}}^{k}$ and $\boldsymbol{S}_{\boldsymbol{q}}^{i, k}, \boldsymbol{S}_{\boldsymbol{u}}^{i, k}$ are defined analogously.

Theorem 3.1 Let $\left\{p_{\tau, h}, \boldsymbol{q}_{\tau, h}\right\} \in \mathcal{W}_{\tau, h}^{r, s} \times \mathcal{V}_{\tau, h}^{r, s}, \boldsymbol{u}_{\tau, h} \in \mathcal{Z}_{\tau, h}^{r, s+1}$ denote the fully discrete space-time $\operatorname{MFEM}(s) d G(s+1)-d G(r)$ approximation of (1.1), (1.2). Let $\left\{p_{\tau, h}^{k}, \boldsymbol{q}_{\tau, h}^{k}, \boldsymbol{u}_{\tau, h}^{k}\right\}$ be defined by (2.7) with coefficients being given by (2.8), (2.9) and (2.10). Then, if the parameter $L>0$ and penalty function $\delta$ in $J_{\delta}$ of (2.10) are chosen sufficiently large, the sequence $\left\{S_{p}^{i, k}\right\}_{k}$, for $i=0, \ldots, r$, converges in $W_{h}$. This implies the convergence of $\left\{p_{\tau, h}^{k}\left(t_{n}^{ \pm}\right), \boldsymbol{q}_{\tau, h}^{k}\left(t_{n}^{ \pm}\right), \boldsymbol{u}_{\tau, h}^{k}\left(t_{n}^{ \pm}\right)\right\}$to $\left\{p_{\tau, h}\left(t_{n}^{ \pm}\right), \boldsymbol{q}_{\tau, h}\left(t_{n}^{ \pm}\right), \boldsymbol{u}_{\tau, h}\left(t_{n}^{ \pm}\right)\right\}$in $W_{h} \times \boldsymbol{V}_{h} \times \boldsymbol{H}_{h}$ for $k \rightarrow \infty$ and $n=1, \ldots, N$, as well as of $p_{\tau, h}^{k}, \boldsymbol{q}_{\tau, h}^{k}$ and $\boldsymbol{u}_{\tau, h}^{k}$ in $L^{2}\left(I_{n} ; W\right)$ and $L^{2}\left(I_{n} ; \boldsymbol{L}^{2}(\Omega)\right)$, respectively.

Proof. We split the proof into several steps.

1. Step (Error equations). By substracting Eqs. 2.8-2.10) from the fully discrete monolithic space-time approximation $\operatorname{MFEM}(s) \mathrm{dG}(s+1)-\mathrm{dG}(r)$ of the Biot system (1.1), (1.2) we obtain for $i=0, \ldots, r$ that

$$
\begin{aligned}
& \frac{1}{M} \sum_{j=0}^{r} \alpha_{i j}\left\langle E_{p}^{j, k+1}, w_{h}\right\rangle+L \sum_{j=0}^{r} \alpha_{i j}\left\langle E_{p}^{j, k+1}-E_{p}^{j, k}, w_{h}\right\rangle \\
& \quad+\tau_{n} \beta_{i i}\left\langle\nabla \cdot \boldsymbol{E}_{\boldsymbol{q}}^{i, k+1}, w_{h}\right\rangle=-b \sum_{j=0}^{r} \alpha_{i j} \sum_{K \in \mathcal{T}_{h}}\left\langle\nabla \cdot \boldsymbol{E}_{\boldsymbol{u}}^{j, k}, w_{h}\right\rangle_{K}, \\
& \left\langle\boldsymbol{K}^{-1} \boldsymbol{E}_{\boldsymbol{q}}^{i, k+1}, \boldsymbol{v}_{h}\right\rangle-\left\langle E_{p}^{i, k+1}, \nabla \cdot \boldsymbol{v}_{h}\right\rangle=0, \\
& \sum_{K \in \mathcal{T}_{h}}\left\langle\boldsymbol{\sigma}\left(\boldsymbol{E}_{\boldsymbol{u}}^{i, k+1}\right), \boldsymbol{\varepsilon}\left(\boldsymbol{z}_{h}\right)\right\rangle_{K}+J_{\delta}\left(\boldsymbol{E}_{\boldsymbol{u}}^{i, k+1}, \boldsymbol{z}_{h}\right)-J_{d}\left(\boldsymbol{E}_{\boldsymbol{u}}^{i, k+1}, \boldsymbol{z}_{h}\right) \\
& \quad=\sum_{K \in \mathcal{T}_{h}} b\left\langle E_{p}^{i, k+1}, \nabla \cdot \boldsymbol{z}_{h}\right\rangle_{k}-b J_{p}\left(E_{p}^{i, k+1}, \boldsymbol{z}_{h}\right)
\end{aligned}
$$

for all $w_{h} \in W_{h}, \boldsymbol{v}_{h} \in \boldsymbol{V}_{h}, \boldsymbol{z}_{h} \in \boldsymbol{H}_{h}$. 
2. Step (Choice of test function in Eq. (3.1)). We test Eq. (3.1) with $w_{h}=$ $\sum_{j=0}^{r} \alpha_{i j} E_{p}^{j, k+1}$. By some calculations following [1, p. 756] we get that

$$
\begin{aligned}
& \left(\frac{1}{M \beta_{i i}}+\frac{L}{2 \beta_{i i}}\right)\left\|S_{p}^{i, k+1}\right\|^{2}+\frac{L}{2 \beta_{i i}}\left\|S_{p}^{i, k+1}-S_{p}^{i, k}\right\|^{2}-\frac{L}{2 \beta_{i i}}\left\|S_{p}^{i, k}\right\|^{2} \\
& +\tau_{n}\left\langle\nabla \cdot \boldsymbol{E}_{\boldsymbol{q}}^{i, k+1}, S_{p}^{i, k+1}\right\rangle=-\frac{b}{\beta_{i i}} \sum_{K \in \mathcal{T}_{h}}\left\langle\nabla \cdot \boldsymbol{S}_{\boldsymbol{u}}^{i, k}, S_{p}^{i, k+1}\right\rangle_{K} .
\end{aligned}
$$

3. Step (Summation of Eq. (3.2) and choice of test function). Changing the index $i$ in Eq. (3.2) to $j$, multiplying this equation with $\alpha_{i j}$, summing up from $j=0$ to $r$ and testing with $\boldsymbol{v}_{h}=\tau_{n} \boldsymbol{E}_{\boldsymbol{q}}^{i, k+1} \in \boldsymbol{V}_{h}$ we have that

$$
\tau_{n}\left\langle\boldsymbol{K}^{-1} \boldsymbol{S}_{\boldsymbol{q}}^{i, k+1}, \boldsymbol{E}_{\boldsymbol{q}}^{i, k+1}\right\rangle-\tau_{n}\left\langle S_{p}^{i, k+1}, \nabla \cdot \boldsymbol{E}_{\boldsymbol{q}}^{i, k+1}\right\rangle=0
$$

Adding Eq. (3.5) to Eq. (3.4) implies that

$$
\begin{aligned}
& \left(\frac{1}{M \beta_{i i}}+\frac{L}{2 \beta_{i i}}\right)\left\|S_{p}^{i, k+1}\right\|^{2}+\frac{L}{2 \beta_{i i}}\left\|S_{p}^{i, k+1}-S_{p}^{i, k}\right\|^{2}-\frac{L}{2 \beta_{i i}}\left\|S_{p}^{i, k}\right\|^{2} \\
& +\tau_{n}\left\langle\boldsymbol{K}^{-1} \boldsymbol{S}_{\boldsymbol{q}}^{i, k+1}, \boldsymbol{E}_{\boldsymbol{q}}^{i, k+1}\right\rangle=-\frac{b}{\beta_{i i}} \sum_{K \in \mathcal{T}_{h}}\left\langle\nabla \cdot \boldsymbol{S}_{\boldsymbol{u}}^{i, k}, S_{p}^{i, k+1}\right\rangle_{K} .
\end{aligned}
$$

4. Step (Summation of Eq. (3.3) and choice of test function). Changing the index $i$ in Eq. (3.3) to $j$, multiplying the resulting equation with $\alpha_{i j}$, summing up from $j=0$ to $r$ and choosing $\boldsymbol{z}_{h}=\beta_{i i}^{-1} \boldsymbol{S}_{\boldsymbol{u}}^{i, k} \in \boldsymbol{H}_{h}$ we find that

$$
\begin{aligned}
& \frac{1}{\beta_{i i}} \sum_{K \in \mathcal{T}_{h}}\left\langle\boldsymbol{\sigma}\left(\boldsymbol{S}_{\boldsymbol{u}}^{i, k+1}\right), \boldsymbol{\varepsilon}\left(\boldsymbol{S}_{\boldsymbol{u}}^{i, k}\right)\right\rangle_{K}+\frac{1}{\beta_{i i}} J_{\delta}\left(\boldsymbol{S}_{\boldsymbol{u}}^{i, k+1}, \boldsymbol{S}_{\boldsymbol{u}}^{i, k}\right)-\frac{1}{\beta_{i i}} J_{d}\left(\boldsymbol{S}_{\boldsymbol{u}}^{i, k+1}, \boldsymbol{S}_{\boldsymbol{u}}^{i, k}\right) \\
& =\frac{b}{\beta_{i i}} \sum_{K \in \mathcal{T}_{h}}\left\langle S_{p}^{i, k+1}, \nabla \cdot \boldsymbol{S}_{\boldsymbol{u}}^{i, k}\right\rangle_{K}-\frac{b}{\beta_{i i}} J_{p}\left(S_{p}^{i, k+1}, \boldsymbol{S}_{\boldsymbol{u}}^{i, k}\right)
\end{aligned}
$$

Adding Eq. (3.7) to Eq. (3.6) leads to

$$
\begin{aligned}
& \left(\frac{1}{M \beta_{i i}}+\frac{L}{2 \beta_{i i}}\right)\left\|S_{p}^{i, k+1}\right\|^{2}+\frac{L}{2 \beta_{i i}}\left\|S_{p}^{i, k+1}-S_{p}^{i, k}\right\|^{2}+\tau_{n}\left\langle\boldsymbol{K}^{-1} \boldsymbol{S}_{\boldsymbol{q}}^{i, k+1}, \boldsymbol{E}_{\boldsymbol{q}}^{i, k+1}\right\rangle \\
& \quad+\frac{1}{\beta_{i i}} J_{\delta}\left(\boldsymbol{S}_{\boldsymbol{u}}^{i, k+1}, \boldsymbol{S}_{\boldsymbol{u}}^{i, k}\right)+\frac{1}{\beta_{i i}} \sum_{K \in \mathcal{T}_{h}}\left\langle\boldsymbol{\sigma}\left(\boldsymbol{S}_{\boldsymbol{u}}^{i, k+1}\right), \boldsymbol{\varepsilon}\left(\boldsymbol{S}_{\boldsymbol{u}}^{i, k}\right)\right\rangle_{K} \\
& =\frac{L}{2 \beta_{i i}}\left\|S_{p}^{i, k}\right\|^{2}-\frac{b}{\beta_{i i}} J_{p}\left(S_{p}^{i, k+1}, \boldsymbol{S}_{\boldsymbol{u}}^{i, k}\right)+\frac{1}{\beta_{i i}} J_{d}\left(\boldsymbol{S}_{\boldsymbol{u}}^{i, k+1}, \boldsymbol{S}_{\boldsymbol{u}}^{i, k}\right)
\end{aligned}
$$

5. Step (Formation of incremental equation for (3.3), summation and choice of test function.) Firstly, we write Eq. (3.3) for two consecutive iterations, $k$ and $k+1$, and substract the resulting equations from each other. Secondly, we change the 
index $i$ in the thus obtained equations to $j$, multiply them with $\alpha_{i j}$ and sum up from $j=0$ to $r$ to obtain that

$$
\begin{aligned}
& \sum_{K \in \mathcal{T}_{h}}\left\langle\boldsymbol{\sigma}\left(\boldsymbol{S}_{\boldsymbol{u}}^{i, k+1}-\boldsymbol{S}_{\boldsymbol{u}}^{i, k}\right), \boldsymbol{\varepsilon}\left(\boldsymbol{z}_{h}\right)\right\rangle_{K}+J_{\delta}\left(\boldsymbol{S}_{\boldsymbol{u}}^{i, k+1}-\boldsymbol{S}_{\boldsymbol{u}}^{i, k}, \boldsymbol{z}_{h}\right)=b \sum_{K \in \mathcal{T}_{h}}\left\langle S_{p}^{i, k+1}\right. \\
& \left.-S_{p}^{i, k}, \nabla \cdot \boldsymbol{z}_{h}\right\rangle_{K}-b J_{p}\left(S_{p}^{i, k+1}-S_{p}^{i, k}, \boldsymbol{z}_{h}\right)+J_{d}\left(\boldsymbol{S}_{\boldsymbol{u}}^{i, k+1}-\boldsymbol{S}_{\boldsymbol{u}}^{i, k}, \boldsymbol{z}_{h}\right)
\end{aligned}
$$

for all $\boldsymbol{z}_{h} \in \boldsymbol{H}_{h}$. Choosing $\boldsymbol{z}_{h}=\boldsymbol{S}_{\boldsymbol{u}}^{i, k+1}-\boldsymbol{S}_{\boldsymbol{u}}^{i, k} \in \boldsymbol{H}_{h}$ in (3.9), dividing by $\beta_{i i}>0$ and summing up the resulting identity from $i=0$ to $r$, we find that

$$
\begin{aligned}
& \sum_{i=0}^{r} \frac{1}{\beta_{i i}}\left(\sum_{K \in \mathcal{T}_{h}}\left\langle\boldsymbol{\sigma}\left(\boldsymbol{S}_{\boldsymbol{u}}^{i, k+1}-\boldsymbol{S}_{\boldsymbol{u}}^{i, k}\right), \boldsymbol{\varepsilon}\left(\boldsymbol{S}_{\boldsymbol{u}}^{i, k+1}-\boldsymbol{S}_{\boldsymbol{u}}^{i, k}\right)\right\rangle_{K}+J_{\delta}\left(\boldsymbol{S}_{\boldsymbol{u}}^{i, k+1}-\boldsymbol{S}_{\boldsymbol{u}}^{i, k}, \boldsymbol{S}_{\boldsymbol{u}}^{i, k+1}\right.\right. \\
& \left.\left.\quad-\boldsymbol{S}_{\boldsymbol{u}}^{i, k}\right)\right)=\sum_{i=0}^{r} \frac{1}{\beta_{i i}}\left(\sum_{K \in \mathcal{T}_{h}} b\left\langle S_{p}^{i, k+1}-S_{p}^{i, k}, \nabla \cdot\left(\boldsymbol{S}_{\boldsymbol{u}}^{i, k+1}-\boldsymbol{S}_{\boldsymbol{u}}^{i, k}\right)\right\rangle_{K}\right. \\
& \left.\quad-b J_{p}\left(S_{p}^{i, k+1}-S_{p}^{i, k}, \boldsymbol{S}_{\boldsymbol{u}}^{i, k+1}-\boldsymbol{S}_{\boldsymbol{u}}^{i, k}\right)+J_{d}\left(\boldsymbol{S}_{\boldsymbol{u}}^{i, k+1}-\boldsymbol{S}_{\boldsymbol{u}}^{i, k}, \boldsymbol{S}_{\boldsymbol{u}}^{i, k+1}-\boldsymbol{S}_{\boldsymbol{u}}^{i, k}\right)\right)
\end{aligned}
$$

Further, from Eq. (3.9) with $\boldsymbol{z}_{h}=\boldsymbol{S}_{\boldsymbol{u}}^{i, k+1}-\boldsymbol{S}_{\boldsymbol{u}}^{i, k}$ we get by means of the inequalities of Cauchy-Schwarz and Cauchy-Young that

$$
\begin{aligned}
& \sum_{K \in \mathcal{T}_{h}}\left\langle\boldsymbol{\sigma}\left(\boldsymbol{S}_{\boldsymbol{u}}^{i, k+1}-\boldsymbol{S}_{\boldsymbol{u}}^{i, k}\right), \boldsymbol{\varepsilon}\left(\boldsymbol{S}_{\boldsymbol{u}}^{i, k+1}-\boldsymbol{S}_{\boldsymbol{u}}^{i, k}\right)\right\rangle_{K}+J_{\delta}\left(\boldsymbol{S}_{\boldsymbol{u}}^{i, k+1}-\boldsymbol{S}_{\boldsymbol{u}}^{i, k}, \boldsymbol{S}_{\boldsymbol{u}}^{i, k+1}\right. \\
& \left.-\boldsymbol{S}_{\boldsymbol{u}}^{i, k}\right) \leq \frac{b^{2}}{2 \lambda}\left\|S_{p}^{i, k+1}-S_{p}^{i, k}\right\|^{2}+\sum_{K \in \mathcal{T}_{h}} \frac{\lambda}{2}\left\|\nabla \cdot\left(\boldsymbol{S}_{\boldsymbol{u}}^{i, k+1}-\boldsymbol{S}_{\boldsymbol{u}}^{i, k}\right)\right\|_{K}^{2} \\
& -b J_{p}\left(S_{p}^{i, k+1}-S_{p}^{i, k}, \boldsymbol{S}_{\boldsymbol{u}}^{i, k+1}-\boldsymbol{S}_{\boldsymbol{u}}^{i, k}\right)+J_{d}\left(\boldsymbol{S}_{\boldsymbol{u}}^{i, k+1}-\boldsymbol{S}_{\boldsymbol{u}}^{i, k}, \boldsymbol{S}_{\boldsymbol{u}}^{i, k+1}-\boldsymbol{S}_{\boldsymbol{u}}^{i, k}\right) .
\end{aligned}
$$

The terms $J_{p}$ and $J_{d}$ can be bounded by means of (cf. [4, p. 429, p. 431])

$$
\begin{aligned}
\left|J_{p}\left(w_{h}, \boldsymbol{z}_{h}\right)\right| & \leq \frac{c}{\delta_{\min }}\left\|w_{h}\right\|^{2}+\frac{1}{R} J_{\delta}\left(\boldsymbol{z}_{h}, \boldsymbol{z}_{h}\right), \\
\left|\sum_{e \in \mathcal{E}_{h}^{\text {int }}}\left\langle\left\{\boldsymbol{\sigma}\left(\boldsymbol{y}_{h}\right) \boldsymbol{\nu}^{e}\right\},\left[\boldsymbol{z}_{h}\right]\right\rangle_{e}\right| & \leq \frac{c}{\delta_{\min }} \sum_{e \in \mathcal{E}_{h}^{\text {int }}}\left\langle\boldsymbol{\sigma}\left(\boldsymbol{y}_{h}\right), \boldsymbol{\varepsilon}\left(\boldsymbol{y}_{h}\right)\right\rangle_{K}+\frac{1}{R} J_{\delta}\left(\boldsymbol{z}_{h}, \boldsymbol{z}_{h}\right)
\end{aligned}
$$

for $R \in \mathbb{N}, R>1$ and some constant $c>0$, such that they can be absorbed by the left-hand side, if the penalty parameter $\delta_{\min }$ is chosen sufficiently large.

6. Step (Summation of Eq. (3.8) and combination with Eq. (3.10)). Using in (3.8) that $4\langle x, y\rangle=\|x+y\|^{2}-\|x-y\|^{2}$, summing up the resulting equation over $i$ 
and using (3.10) together with (3.12) ) we get that

$$
\begin{aligned}
& \sum_{i=0}^{r}\left\{\left(\frac{1}{M \beta_{i i}}+\frac{L}{2 \beta_{i i}}\right)\left\|S_{p}^{i, k+1}\right\|^{2}+\frac{L}{2 \beta_{i i}}\left\|S_{p}^{i, k+1}-S_{p}^{i, k}\right\|^{2}\right. \\
& \left.+\tau_{n}\left\langle\boldsymbol{K}^{-1} \boldsymbol{S}_{\boldsymbol{q}}^{i, k+1}, \boldsymbol{E}_{\boldsymbol{q}}^{i, k+1}\right\rangle\right\}+\sum_{i=0}^{r} \frac{1}{4 \beta_{i i}} J_{\delta}\left(\boldsymbol{S}_{\boldsymbol{u}}^{i, k+1}+\boldsymbol{S}_{\boldsymbol{u}}^{i, k}, \boldsymbol{S}_{\boldsymbol{u}}^{i, k+1}+\boldsymbol{S}_{\boldsymbol{u}}^{i, k}\right) \\
& +\sum_{i=0}^{r} \frac{1}{4 \beta_{i i}} \sum_{K \in \mathcal{T}_{h}}\left\langle\boldsymbol{\sigma}\left(\boldsymbol{S}_{\boldsymbol{u}}^{i, k+1}+\boldsymbol{S}_{\boldsymbol{u}}^{i, k}\right), \boldsymbol{\varepsilon}\left(\boldsymbol{S}_{\boldsymbol{u}}^{i, k+1}+\boldsymbol{S}_{\boldsymbol{u}}^{i, k}\right)\right\rangle_{K} \leq \sum_{i=0}^{r} \frac{L}{2 \beta_{i i}}\left\|S_{p}^{i, k}\right\|^{2} \\
& +\sum_{i=0}^{r} \frac{1}{4 \beta_{i i}} \sum_{K \in \mathcal{T}_{h}} b\left\langle S_{p}^{i, k+1}-S_{p}^{i, k}, \nabla \cdot\left(\boldsymbol{S}_{\boldsymbol{u}}^{i, k+1}-\boldsymbol{S}_{\boldsymbol{u}}^{i, k}\right)\right\rangle_{K}+c \sum_{i=0}^{r} \frac{b^{2}}{\beta_{i i}} \| S_{p}^{i, k+1} \\
& -S_{p}^{i, k} \|^{2}-\sum_{i=0} \frac{1}{\beta_{i i}}\left(b J_{p}\left(S_{p}^{i, k+1}, \boldsymbol{S}_{\boldsymbol{u}}^{i, k}\right)-J_{d}\left(\boldsymbol{S}_{\boldsymbol{u}}^{i, k+1}, \boldsymbol{S}_{\boldsymbol{u}}^{i, k}\right)\right) .
\end{aligned}
$$

For the second term $T_{2}$ on the right-hand side, (3.11) and (3.12) yield that

$$
\left|T_{2}\right| \leq c \sum_{i=0}^{r} \frac{b^{2}}{\lambda \beta_{i i}}\left\|S_{p}^{i, k+1}-S_{p}^{i, k}\right\|^{2} .
$$

On the left-hand side, the third term can be rewritten by [1, p. 760, Eq. (4.29)]. The fourth and fifth term are rewritten by first using the identity $2\langle x, y\rangle=\langle x, x\rangle+\langle y, y\rangle-$ $\langle x-y, x-y\rangle$ and then applying (3.11) with (3.12).

7. Step (Contraction). We combine the results of the 5 th and 6 th step to

$$
\begin{aligned}
& \sum_{i=0}^{r}\left\{\left(\frac{1}{M \beta_{i i}}+\frac{L}{2 \beta_{i i}}\right)\left\|S_{p}^{i, k+1}\right\|^{2}+\frac{L}{2 \beta_{i i}}\left\|S_{p}^{i, k+1}-S_{p}^{i, k}\right\|^{2}\right\} \\
& \quad+\frac{\tau_{n}}{2}\left\|\boldsymbol{K}^{-1 / 2} \boldsymbol{e}_{\boldsymbol{q}}^{k+1}\left(t_{n}^{-}\right)\right\|^{2}+\frac{\tau_{n}}{2}\left\|\boldsymbol{K}^{-1 / 2} \boldsymbol{e}_{\boldsymbol{q}}^{k+1}\left(t_{n-1}^{+}\right)\right\|^{2} \\
& \quad+\sum_{i=0}^{r} \frac{1}{2 \beta_{i i}} \sum_{K \in \mathcal{T}_{h}}\left\langle\boldsymbol{\sigma}\left(\boldsymbol{S}_{\boldsymbol{u}}^{i, k+1}\right), \boldsymbol{\varepsilon}\left(\boldsymbol{S}_{\boldsymbol{u}}^{i, k+1}\right)\right\rangle_{K}+\sum_{i=0}^{r} \frac{1}{2 \beta_{i i}} J_{\delta}\left(\boldsymbol{S}_{\boldsymbol{u}}^{i, k+1}, \boldsymbol{S}_{\boldsymbol{u}}^{i, k+1}\right) \\
& \quad+\sum_{i=0}^{r} \frac{1}{2 \beta_{i i}} \sum_{K \in \mathcal{T}_{h}}\left\langle\boldsymbol{\sigma}\left(\boldsymbol{S}_{\boldsymbol{u}}^{i, k}\right), \boldsymbol{\varepsilon}\left(\boldsymbol{S}_{\boldsymbol{u}}^{i, k}\right)\right\rangle_{K}+\sum_{i=0}^{r} \frac{1}{2 \beta_{i i}} J_{\delta}\left(\boldsymbol{S}_{\boldsymbol{u}}^{i, k}, \boldsymbol{S}_{\boldsymbol{u}}^{i, k}\right) \\
& \leq \sum_{i=0}^{r} \frac{L}{2 \beta_{i i}}\left\|S_{p}^{i, k}\right\|^{2}+c \sum_{i=0}^{r} \frac{b^{2}}{\lambda \beta_{i i}}\left\|S_{p}^{i, k+1}-S_{p}^{i, k}\right\|^{2} \\
& \quad-\sum_{i=0}^{r} \frac{b}{\beta_{i i}} J_{p}\left(S_{p}^{i, k+1}, \boldsymbol{S}_{\boldsymbol{u}}^{i, k}\right)+\sum_{i=0}^{r} \frac{1}{\beta_{i i}} J_{d}\left(\boldsymbol{S}_{\boldsymbol{u}}^{i, k+1}, \boldsymbol{S}_{\boldsymbol{u}}^{i, k}\right) .
\end{aligned}
$$

Using (3.12) the last two terms on the right-hand side of (3.14) can be absorbed by terms on the left-hand side, if $L>0$ and the penalty function $\delta$ in $J_{\delta}$ of (2.10) are chosen sufficiently large, i.e. $L>c b^{2} / \lambda$. Inequality (3.14) then shows the convergence of the iterates $S_{p}^{i, k}$ in $W_{h}$, for $i=0, \ldots, r$. From (3.14) along with the convergence of 
$S_{p}^{i, k}$ we get the convergence of $\boldsymbol{e}_{\boldsymbol{q}}^{k}\left(t_{n}^{-}\right)$and $\boldsymbol{e}_{\boldsymbol{q}}^{k}\left(t_{n-1}^{+}\right)$to $\mathbf{0}$ for $k \rightarrow \infty$. Eq. (3.2) together with the convergence of $\boldsymbol{e}_{\boldsymbol{q}}^{k}\left(t_{n}^{-}\right)$yields the convergence of $e_{p}^{k}\left(t_{n}^{-}\right)$to 0 for $k \rightarrow \infty$. Finally, Eq. (3.3) implies the convergence of $\boldsymbol{e}_{\boldsymbol{u}}^{k}\left(t_{n}^{-}\right)$to $\mathbf{0}$. The rest follows as in 1 , Cor. 4.6].

\section{References}

[1] M. Bause, F. Radu, U. Köcher, Space-time finite element approximation of the Biot poroelasticity system with iterative coupling, Comput. Methods Appl. Mech. Engrg., 320 (2017), 745-768.

[2] J. W. Both, M. Borregales, J. M. Nordbotton, K. Kundan, F. A. Radu, Robust fixed stress splitting for Biot's equations in heterogeneous media, Appl. Math. Lett., 68 (2017), 101-108.

[3] A. Mikelić, M. Wheeler, Convergence of iterative coupling for coupled flow and geomechanics, Comput. Geosci., 17 (2013), 479-496.

[4] P. Philips, M. Wheeler, A coupling of mixed and discontinuous Galerkin finite element methods for poroelasticity, Comput. Geosci., 12 (2008), 417-435.

[5] P. Philips, M. Wheeler, Overcoming the problem of locking in linear elasticity and poroelasticity: an heuristic approach, Comput. Geosci., 13 (2009), 5-12.

[6] S.-Y. Yi, A study of two modes of locking in poroelasticity, SIAM J. Numer. Anal., 55 (2017), 1915-1936. 